\title{
Evaluation of Radiofrequency Catheter Ablation Settings for Variable Atrial Tissue Depth and Flow Conditions
}

\author{
Desmond Dillon-Murphy ${ }^{1}$, David Nordsletten ${ }^{1}$, Navjeevan Soor $^{2}$, Henry Chubb $^{1}$, Mark O’Neill ${ }^{1}$, \\ Adelaide de Vecchi ${ }^{1}$, Oleg Aslanidi ${ }^{1}$ \\ ${ }^{1}$ Department of Biomedical Engineering, King's College London, London, United Kingdom \\ ${ }^{2}$ Department of Bioengineering, Imperial College London, London, United Kingdom
}

\begin{abstract}
Catheter ablation (CA) is a potentially curative therapy for paroxysmal atrial fibrillation, however up to $50 \%$ of patients may require a repeat procedure to achieve the best results. While this may be due to insufficient radiofrequency $(R F)$ energy delivery to achieve transmural, contiguous ablation lesions (TAL), excessive RF application may result in extracardiac injury and complications. We aim to evaluate optimal RFCA temperature settings for variable atrial tissue depth and blood flow conditions.

Heat spread in atrial tissue and blood was simulated using the Pennes bioheat equation with an additional convective term in the blood. The minimum catheter contact time required for TAL formation increased with increasing either atrial wall thickness (AWT) or laminar flow velocity (LFV). Thus, during ablation of atrial tissue regions with AWT of 1 and $5.5 \mathrm{~mm}$ and $L F V$ of $0.1 \mathrm{~m} / \mathrm{s}$, TALs were formed in 12 and $153 \mathrm{~s}$, respectively. When LFV was increased to $1.2 \mathrm{~m} / \mathrm{s}$, the TAL formation time increased to 21 and $174 \mathrm{~s}$. The maximum temperature in the blood volume stayed below the coagulation risk threshold of $70^{\circ} \mathrm{C}$ for all $A W T$ values with LFVs over $0.6 \mathrm{~m} / \mathrm{s}$, but raised above the threshold for $L F V \leqslant 0.6 \mathrm{~m} / \mathrm{s}$ and $A W T>1.5 \mathrm{~mm}$.

This study demonstrates the potential for optimizing RFCA settings according to atrial tissue depth and flow, which may improve the efficacy of RFCA therapy.
\end{abstract}

\section{Introduction}

Atrial fibrillation (AF) is the most common cardiac arrhythmia, affecting over 30 million people worldwide [1]. It is characterised by irregular, rapid electrical activations of the atria and carries a high risk of heart failure and stroke. Radiofrequency catheter ablation (RFCA) is gradually supplanting antiarrhythmic drug therapy as a front-line treatment for AF termination. This procedure delivers RF energy via a catheter to produce produce electrically inert, transmural lesions in the myocardium to isolate or remove areas contributing to the generation or maintenance of the activity sustaining AF (e.g., drivers near the pulmonary veins, PVs) [2]. In the absence of new electrical drivers, reoccurrence of AF may be due to gaps between lesions or a lack of transmurality, which both prevent the complete isolation of the drivers [3].

However, RFCA procedures are not always effective. The success rates for single-procedure PV isolations in paroxysmal AF patients are only 38-70\% [1]. In persistent $\mathrm{AF}, \mathrm{PV}$ isolation alone is not sufficient and further areas of the atrial wall are targeted. Moreover, in $4.5 \%$ of patients RFCA procedures can result in major complications[4]. In-vitro studies also suggest that in regions with high blood flow rate and convective cooling, e.g., above the mitral valve, more power must be supplied to achieve transmural lesions, resulting in higher localised temperature, overheating atrial tissue and producing larger lesions $[5,6]$. This excessive ablation can cause permanent loss of contractility outside of target areas and heart failure [7].

As blood flow rates vary considerably inside the atria, the lesion size is hard to predict. Additionally, high interand intra- patient variations in atrial wall thickness (AWT) [8] presents a major issue when creating the transmural lesions required for effective RFCA treatments. Knowledge of both AWT and blood flow rates could provide clinicians with useful information to optimise the ablation procedure - by using the minimal amount of RF energy and catheter contact time to achieve lesion transmurality. Furthermore, such knowledge could also reduce the risk of complications. For example, PV stenosis can be avoided by minimising catheter contact time at target areas to ensure temperatures stay below risk levels [9].

This study aims to apply computational modelling [10, 11] to simulate RFCA in realistic atrial blood flow and wall thickness settings. This will provide novel information on the minimal catheter contact time required to fully form transmural ablation lesions (TAL) - while also assessing the risks of complications - for specific flow and AWT. The model predictions could potentially be used to improve the efficacy and safety of RFCA procedures in the clinic. 


\section{Methods}

\subsection{Thermal model with convection}

To simulate the temperature distributions in atrial tissue and blood the Pennes bioheat equation with an additional convective term in the blood was used [11]:

$$
c \frac{\partial T}{\partial t}=D \nabla^{2} T+Q_{c a t h}-\vec{v} \cdot \nabla T
$$

where

$$
\begin{aligned}
D & =k / \rho c \\
Q_{c a t h} & =\vec{E} \cdot \vec{J}=\sigma(\nabla V)^{2} \\
\vec{E} & =\nabla V \\
\vec{J} & =\sigma \nabla V
\end{aligned}
$$

and the voltage is determined from the Laplace's equation:

$$
\nabla^{2} V=0
$$

The variables in the equations above are as follows:

$c \quad$ heat capacity of the myocardium $(\mathrm{J} /(\mathrm{kg} \cdot \mathrm{K}))$

$T$ temperature of the blood or myocardium (K)

$D \quad$ thermal diffusivity $\left(\mathrm{m}^{2} / \mathrm{s}\right)$

$Q_{\text {cath }}$ heat applied by the catheter $\left(\mathrm{W} / \mathrm{m}^{3}\right)$

$\vec{v} \quad$ blood velocity ( $\mathrm{m} / \mathrm{s})$

$k \quad$ thermal conductivity $(\mathrm{W} /(\mathrm{m} \cdot \mathrm{K}))$

$\rho \quad$ density $\left(\mathrm{kg} / \mathrm{m}^{3}\right)$

$\sigma \quad$ electric conductivity $(\mathrm{S} / \mathrm{m})$

$\vec{E} \quad$ electrical field intensity $(\mathrm{V} / \mathrm{m})$

$\vec{J} \quad$ current density $\left(\mathrm{A} / \mathrm{m}^{2}\right)$

Heat loss due to perfusion in the myocardium was neglected as nominal relative to the heat applied during ablation. The Arrhenius method was used to evaluate tissue thermal injury leading to the TAL formation:

$$
\Omega(t)=\int_{0}^{t} A \cdot e^{\frac{-\Delta E}{R T}} d t=\ln \left[\frac{c(0)}{c(t)}\right]
$$

where $\Omega(t)$ is interpreted as the logarithmic ratio of undamaged tissue at time 0 and ratio of damaged tissue at time $t ; A$ is the rate factor measuring collision $\left(\mathrm{s}^{-1}\right) ; \Delta E$ is the energy barrier the tissue must exceed to be denatured $(\mathrm{J} / \mathrm{mol})$ and $R$ is universal gas constant $(8.3134 \mathrm{~J} / \mathrm{mol} \cdot \mathrm{K})$. If $\Omega(t)=1$ this indicates $63 \%$ probability of irreversible tissue damage and $\Omega(t)>4.3$ indicates $99 \%$ probability.

Equations were solved using the finite difference method on a uniform 3D rectangular domain measuring $50 \times 100 \times 50$ voxels with a spatial step of $0.25 \mathrm{~mm}$. The tissue slab measured $50 \times 100 \times n / 0.25$, where $n$ represented slab thickness in $\mathrm{mm}$, and the remainder of the domain represented blood. The temporal step was set at 5 ms. Throughout the domain an initial temperature was set at $37{ }^{\circ} \mathrm{C}(310.15 \mathrm{~K})$ and a uniform potential set at $0 \mathrm{~V}$.

Values for the constants were determined from literature used for both blood [12] and myocardium (see Table 1). Blood was assumed to have a haematocrit of $40 \%$.

Table 1. Constants used in the model

\begin{tabular}{cccc}
\hline \hline & $\boldsymbol{\rho}$ & $\boldsymbol{c}$ & $\boldsymbol{k}$ \\
& $\mathrm{kg} \cdot \mathrm{m}^{-3}$ & $\mathrm{~J} / \mathrm{kg} \cdot \mathrm{K}$ & $\mathrm{W} / \mathrm{m} \cdot \mathrm{K}$ \\
Tissue & 1,200 & 3,200 & 0.55 \\
Blood & 1,060 & 3,947 & 0.57 \\
\hline \hline & $\boldsymbol{\sigma}$ & $\boldsymbol{A}$ & $\boldsymbol{\Delta} \boldsymbol{E}$ \\
& $\mathrm{S} / \mathrm{m}$ & $\mathrm{s}^{-1}$ & $\mathrm{~J} / \mathrm{mol}$ \\
\hline Tissue & 0.222 & $1.28 \times 10^{22}$ & 145,000 \\
\hline
\end{tabular}

\subsection{Ablation simulation settings}

To explore the relationship between atrial wall depth, blood flow velocity and TAL formation, a series of atrial tissue slabs with blood volume (see Figure 1) were created with AWT defined between 1.0-5.5 mm [8] in steps of 0.5 $\mathrm{mm}$. For each slab, a series of simulations was performed with a uniform laminar flow velocity (LFV) specified in the blood and varied between $0.0-1.2 \mathrm{~m} / \mathrm{s}$ [13] in steps of $0.2 \mathrm{~m} / \mathrm{s}$. In each simulation, an initial voltage of $10 \mathrm{~V}$ was applied to the catheter positioned in the bloodstream on the endocardial surface of the tissue. The simulation continued until some point of the myocardium on the opposite, epicardial surface reached a value of $\Omega \geq 1$, indicating the creation of a transmural lesion through the atrial wall.

\section{Results}

Figure 1 illustrates ablation simulations in 3D atrial slabs with varying AWT under the blood with varying LFV, with the catheter tip positioned at the tissue-blood interface. Both temperature (left panels) and the $\Omega$-value (right panels) representing tissue damage are mapped, and regions where $\Omega \geq 1$ (corresponding to scar tissue forming the lesion) are highlighted. The extremes of the varying LFV are illustrated - a stagnant $0.0 \mathrm{~m} / \mathrm{s}$ flow to the highest simulated velocity of $1.2 \mathrm{~m} / \mathrm{s}$ in steps of $0.2 \mathrm{~m} / \mathrm{s}$ - for three representative AWT values, $1.0 \mathrm{~mm}, 3.5 \mathrm{~mm}$ and $5.0 \mathrm{~mm}$.

Figure 1 shows that blood flow had a significant impact on the shape of ablation lesions. At zero velocity, the lesion formed in a hemisphere like shape evenly around the region in contact with the catheter tip. This was due to the even spreading of heat around the ablation contact area. When exposed to the flow, the temperature distribution changed, with the higher temperatures being downstream of the catheter tip. This change in the temperature distribution causes the lesion to form as a semi-ellipsoid with 


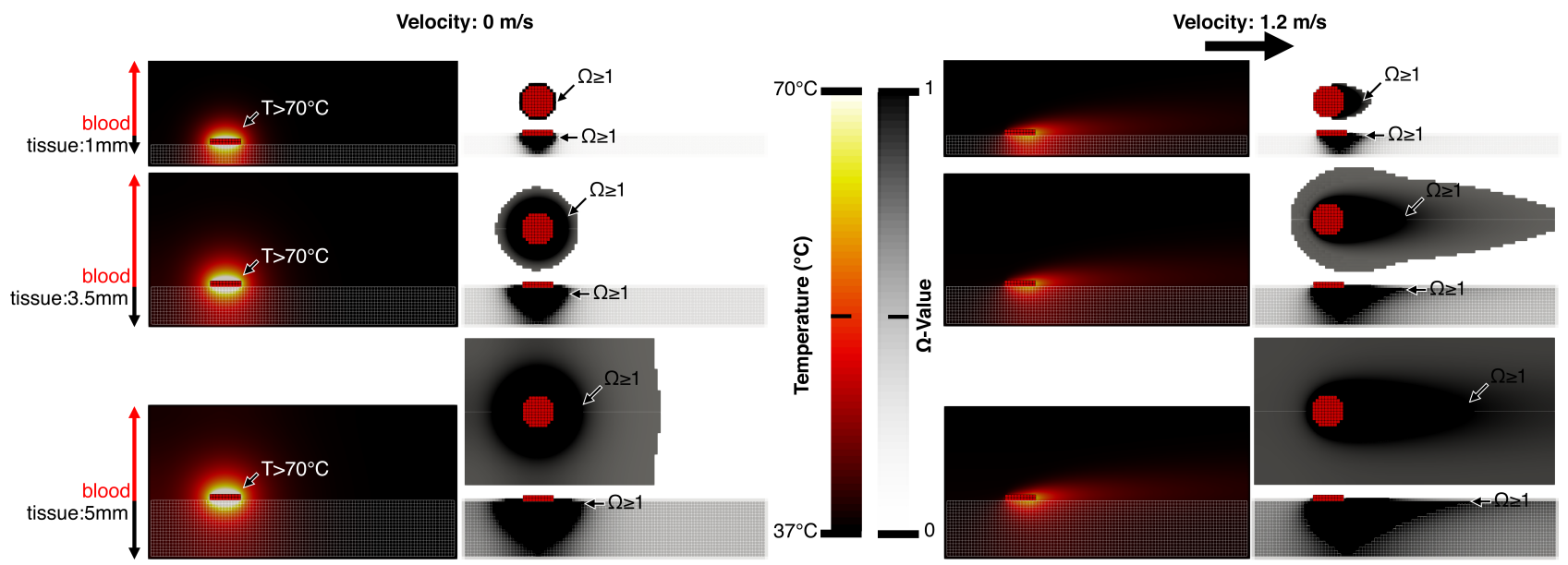

Figure 1. Temperature distribution in atrial tissue and blood: The figure shows temperature distributions in transmural cross sections through 3D atrial slabs under the blood volume (left panels), and the respective cross sections and top down projections of the $\Omega$-value (right panels) in the tissue for AWT of $1.0 \mathrm{~mm}$ (top), $3.5 \mathrm{~mm}$ (middle) and $5.0 \mathrm{~mm}$ (bottom) and LBF of $0.0 \mathrm{~m} / \mathrm{s}$ (left) and $1.2 \mathrm{~m} / \mathrm{s}$ (right). The position of the catheter tip (red) for each simulation is shown. The regions where $\Omega \geq 1$ indicating a $63 \%$ chance of irreversible tissue damage are highlighted (black). The regions where temperature was recorded being $\geq 70{ }^{\circ} \mathrm{C}$ also are highlighted (white) due to the additional risk of blood clot formation.

the long axis in the direction of flow. The point which first reaches $\Omega \geq 1$ on the opposing side of the atrial wall also changes, shifting from being directly opposite the midpoint of the catheter tip to being at a point downstream.

Figure 1 also shows that higher LFV tended to be associated with lower blood temperatures, and blood was observed to heat to higher temperatures when LFV was low. The figure highlights regions above $70^{\circ} \mathrm{C}$, which have been associated with a higher likelihood of blood clot formation during the ablation process [14].

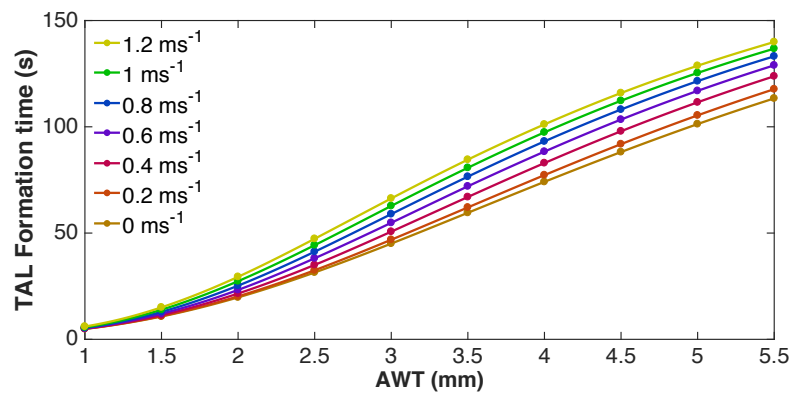

Figure 2. Effect of AWT and LFV on the time to full TAL formation: This was measured as the time when $\Omega$ reached a value $\geq 1$ on the epicadial side of the atrial wall - oppose the catheter tip applied on the endocardium. Here and below, each line represents a different blood velocity.

Figure 2 shows the time to TAL formation (full lesion extending from endocardium to epicardium) against AWT for each value of velocity from $0.0 \mathrm{~m} / \mathrm{s}$ to $1.2 \mathrm{~m} / \mathrm{s}$. The figure suggests that higher flow velocities and thicker atrial wall corresponded to longer times to TAL formation, with the the formation time showing a stronger dependence on the AWT. Note that percentage change in the TAL formation time was similar for AWT values of 1 and $5 \mathrm{~mm}$ : in both cases, the lesion took $23 \%$ longer to form when LFV was $1.2 \mathrm{~m} / \mathrm{s}$ rather than zero. The greatest change was seen for AWT of $2.5 \mathrm{~mm}$, where the difference in TAL formation time was $50.38 \%$ between LFV of 0.0 and $1.2 \mathrm{~m} / \mathrm{s}$. This result suggests that at such intermediate AWT values blood flow has a greater influence on lesion formation.

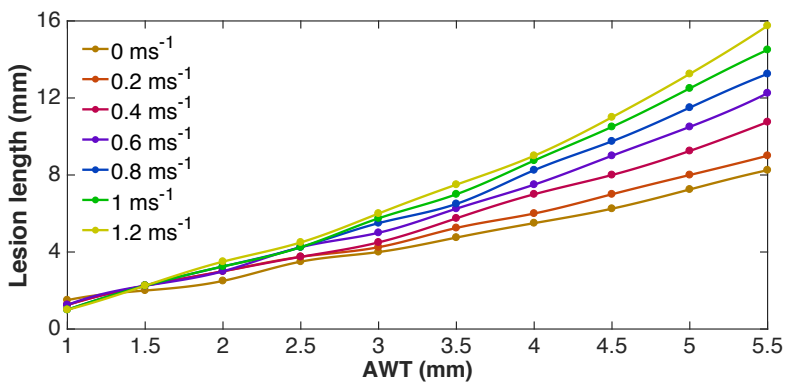

Figure 3. Effect of AWT and LFV on the lesion length: The figure shows length of the long axis of the lesion. Voxels along this axis with $\Omega \geq 1$ were those sampled.

Figure 3 shows the effect varying AWT and blood velocity had on the length of the resulting lesion, with higher velocities associated with longer lesions.

Figure 4 shows the effect varying AWT and LFV had on the blood maximum temperature. The figure suggests that the peak temperatures are observed in low blood velocities 


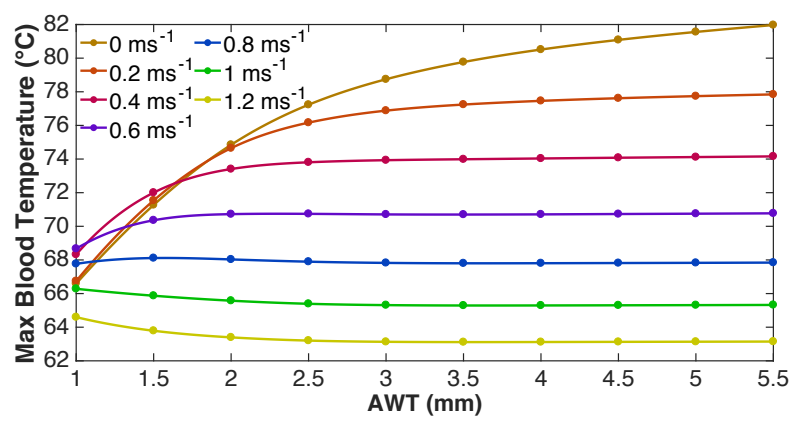

Figure 4. Effect of AWT and LFV on the maximum blood temperature: The figure shows the maximum temperature recorded in the blood for each simulation.

and higher AWT cases. Interestingly, at $1 \mathrm{~mm}$ AWT the highest blood temperatures are recorded at an intermediate LFV of $0.6 \mathrm{~m} / \mathrm{s}$. The reason for the peak temperature not being observed at $0.0 \mathrm{~m} / \mathrm{s}$, as seen for greater AWT, is due to the faster speed of the TAL formation for lower AWT and LFV which does not allow the blood time to heat up.

\section{Discussion}

We developed a novel computational approach to evaluate RFCA settings for realistic atrial tissue depth and flow conditions. For the catheter voltage of $10 \mathrm{~V}$ (corresponding to RF power of $70 \mathrm{~W}$ ), the TAL formation time required for achieving effective TALs increased quasilinearly with both increasing AWT and LFV, with a faster rate of increase for AWT. Both the TAL formation time (Figure 2) and length (Figure 3) can change 10-fold between the condition of low and high AWT and LFV. However, these minimal TAL formation times were generally shorter than catheter contact times in common use. Interestingly, the blood flow had a greater influence on the TAL formation at intermediate AWT values of $2.5 \mathrm{~mm}$, which are average values seen the atrial walls [8]. Risks of the heat-induced blood coagulation due to temperature $\geq 70$ ${ }^{\circ} \mathrm{C}$ [14] also varied with AWT and LFV (Figure 4).

Limitations of the study include the consideration of only simple 3D slab geometries and laminar flow conditions. Future studies will focus on combining the current approach with anatomically detailed 3D atrial models [10] and accurate 3D blood flow simulations [15].

In summary, this study demonstrates the potential for optimizing RFCA settings according to variable atrial tissue depth and flow conditions, which can help improve the safety and efficacy of RFCA therapy in the future.

\section{References}

[1] Camm A, et al. 2012 focused update of the guidelines for the management of atrial fibrillation. Eur Heart J 2013;
34(10):790-790.

[2] O'Neill MD, et al. Catheter ablation for atrial fibrillation. Circulation 2007;116(13):1515-1523.

[3] Katritsis D, et al. Clinical outcome of left atrial ablation for paroxysmal atrial fibrillation is related to the extent of radiofrequency ablation. J Cardiovasc Electrophysiol 2008; 22(1):31-37.

[4] Cappato R, et al. Updated worldwide survey on the methods, efficacy, and safety of catheter ablation for human atrial fibrillation. Circ Arrhythm Electrophysiol 2009; 3(1):32-38.

[5] Cao H, et al. Flow effect on lesion formation in RF cardiac catheter ablation. IEEE Trans Biomed Eng 2001; 48(4):425-433.

[6] Tungjitkusolmun $S$, et al. Guidelines for predicting lesion size at common endocardial locations during radiofrequency ablation. IEEE Trans Biomed Eng 2001; 48(2):194-201.

[7] Lemola K, et al. Effect of left atrial circumferential ablation for atrial fibrillation on left atrial transport function. Heart Rhythm 2005;2(9):923-928.

[8] Varela M, et al. Novel MRI technique enables non-invasive measurement of atrial wall thickness. IEEE Trans Med Imaging 2017;36(8):1607-1614.

[9] Kok L, et al. Effect of heating on pulmonary veins. J Cardiovasc Electrophysiol 2003;14(3):250-254.

[10] Morgan R, Colman MA, Chubb H, Seemann G, Aslanidi OV. Slow conduction in the border zones of patchy fibrosis stabilizes the drivers for atrial fibrillation: Insights from multi-scale human atrial modeling. Front Physiol 2016;7.

[11] Gonzalez-Suarez A, Berjano E. Comparative analysis of different methods of modeling the thermal effect of circulating blood flow during RF cardiac ablation. IEEE Trans Biomed Eng 2016;63(2):250-259.

[12] Duck FA. Thermal Properties of Tissue. Elsevier, 1990. ISBN 978-0-12-222800-1.

[13] Hoit BD. Left atrial size and function: Role in prognosis. J Am Coll Cardiol 2014;63(6):493-505.

[14] Wittkampf F, Nakagawa H. RF catheter ablation: Lessons on lesions. Pacing Clin Electrophysiol 2006;29(11):12851297.

[15] de Vecchi A, et al. A novel methodology for personalized simulations of ventricular hemodynamics from noninvasive imaging data. Comput Med Imaging Graph 2016;51(1):20 31.

\section{Acknowledgements}

This research is funded by grants from the British Heart Foundation (PG/10/69/28524) and EPSRC (EP/P013228/1).

Address for correspondence:

Oleg Aslanidi

Department of Biomedical Engineering, King's College London, St Thomas' Hospital, London SE1 7EH, United Kingdom E-mail: oleg.aslanidi@kcl.ac.uk 